\title{
Research on Marketing Mix of Mobile Online Games -- Honor of Kings
}

\author{
Shurui $\mathrm{Du}{ }^{1, \dagger}$ Xiao Peng ${ }^{2, *}{ }^{*}{ }^{\dagger}$ Zhixia Zhang ${ }^{3, \dagger}$ \\ ${ }^{1}$ Wuhan Britain-China School, 430000, Wuhan, China \\ ${ }^{2}$ Josephine Butler College, Durham, DH1 3DF, Durham, England \\ ${ }^{3}$ Beijing No.80 High School, 100102, Beijing, China \\ *Corresponding author. Email: cfcw48@dur.ac.uk \\ ${ }^{t}$ These authors contributed equally.
}

\begin{abstract}
Due to the rapid development of the Internet, the mobile game industry is one of the most promising and competitive fields for start-ups on the Internet. As people's living standards have improved, the field has attracted a large number of game users. Among many games, Honor of Kings is one of the most popular and representative games, and the study of this game is of great help to the analysis of the entire game marketing. When discussing mobile online marketing strategies, this article referred to some investigation and analysis of some past scholars. At the same time, this article uses a comparative method, literature research, and case study to analyze mobile online game marketing strategies. In addition, this article takes a new approach to the topic and discusses it more deeply and differently than previous articles. In the result part, this article discussed the development status of the mobile online games industry, which included the scale of development and game player group; the current state of marketing in the Mobile Online Games industry -Honor of Kings as an example, including the status quo of Product Strategy, pricing strategy based on consumer psychology, service marketing and platform selection and holiday discounts and promotions; problems with the marketing strategy of the Mobile Online Games industry, including downplaying the role of advertising, inadequate in punishment mechanism, an imperfection in-game experience and bugs and weakness in the anti-addiction(teenager protection) system. In the discussion part, we made industry marketing strategy optimization based on the existing problem. They are online and offline promotion, focusing on bug fixes and enriching gameplay to improve the user experience, improving in-game management and user experience and improving the anti-addiction system. This article could lead to progress in mobile online games and can provide important business value for the development of the industry.
\end{abstract}

Keywords: Honor of kings, Mobile online games, Problems, Marketing mix.

\section{INTRODUCTION}

In the modern network era of rapid development, the mobile game industry is one of the most promising entrepreneurial fields on the Internet. Therefore, with the increasing improvement of people's living standards, the domestic online game culture market is also developing rapidly, and the market for this industry is facing fierce competition. Even if the domestic mobile game started later than foreign countries but did not affect the rapid development of the domestic mobile game industry. According to the data survey, the number of mobile game users in China has reached 507 million, up 3.6\% year on year, and the number of domestic users on active mobile devices reached 1.17 billion by the first half of 2017 [1].
The overall trend of the mobile gaming industry is changing dramatically, which is conducive to growing the number and size of users. Therefore, mobile network games have become the mainstay of the Internet economy, complete the immature industry to mature growth.

According to Li's study, in the mobile game market, although the industry has brought more game choices to everyone, designers and practitioners' key to success is how to design games to attract more users. Therefore, the choice of product marketing strategy is essential. Crossover marketing is about organizing and permeating the commonalities that arise across different industries and products to highlight an emerging attitude and aesthetic for mobile games [2]. For example, the game products 
and communications industry hold traffic marketing activities, and the film and television entertainment crossover cooperation, including video implantation and celebrity endorsement. This kind of cross-industry cooperative marketing expands the popularity of game software and shows the mainstream marketing way of most industries in the future. The concept of brand marketing is reflected in the mobile game industry by some means to imprint the image of the game products behind the enterprise in users' hearts. In other words, when faced with products from both companies, consumers will mostly choose the one they know. If the brand advantage of some successful game product operators can be used to promote new products, user engagement and recognition will have a significant advantage in the game market. Furthermore, Yao and Liu analyzed that advertising is the most traditional and direct and effective marketing strategy in online games. Scenes and characters in the game are integrated into consumers' life scenes by advertisements as a starting point, which makes the game closer to life and easier to attract consumers' attention and participation [1]. Moreover, social marketing mentioned in the research is also closely related to mobile online games. Social marketing starts by identifying the target audience and using their influence to spread the core values inherent in the game. Most games are aimed at young people between the ages of 18 and 30, who know a lot about mobile games and have enough time and money to embrace and play them. Some game companies seize these target consumer groups and let them publicize on prominent social media such as Weibo and WeChat in-game tasks, which can help companies save costs in publicity work, expand the consumer market, and open the popularity of game products. Chaffey and Ellis-Chadwick pointed out that the pricing model in the marketing mix model is in the form of payment to set the product or service [3]. For a game company, choosing the right pricing strategy will maximize the company's profits and balance the expectations of the mobile game organization in the target market. Liu found that mobile games' clothing and item production costs are low, profit margins can be controlled above $90 \%$, and pricing based on volume can increase user acceptance and maximize profits [4]. If the target audience is high-end customization, the focus should be on improving the product positioning and gameplay experience. Therefore, the marketing strategy needs to choose the appropriate and reasonable pricing strategy according to the game market and product positioning. In short, in addition to the high quality of the game itself, the success of a game product is also related to a reasonable marketing strategy. In the future mobile game, the market will face a fiercer competition environment; online game marketing will also become a vital driving force to promote the development of the mobile online game industry. In 2021, users in the Chinese mobile market have reached 10 million.
According to the above literature review, most articles mainly study the marketing strategy of mobile online games. In contrast, few scholars have studied the way Honor of Kings is marketed in mobile games. Therefore, this paper analyzes the development status of the mobile online game industry by using the research method of secondary data and takes the Honor of king's game as an example to reflect the marketing status of the mobile game industry. Secondly, it exposes the problems existing in the marketing strategy of the sales game industry and puts forward reasonable suggestions to optimize these problems.

\section{METHOD}

While discussing strategies of mobile online marketing, this article started in a broad way and referred to some investigation and analysis of some past scholars. This article uses a comparative method, literature research, and case study to analyze mobile online game marketing strategies. Moreover, this article has a new method compared to the past articles, and it discussed this topic more deeply and differently. Thus, this can progress in current research.

\subsection{Comparative Method}

The comparative method, the main body by innate factor endowment or acquired learning innovation, forms a higher value-added comparative advantage, including comparative competitive advantage and comparative cooperation advantage.

In the first part, we come to a horizontal comparison. The horizontal comparison refers to the comparison of two or more things of the same kind. For example, the horizontal comparison between country and country, region and region. According to $\mathrm{Xu}$ [5]'s study, he compared the top 10 best-selling mobile games of 2016. The Honor of Kings topped the list by a landslide with more than $78 \%$ of voters, while other games such as Crossfire and Clash Royale were overtaken by it with $32 \%$ and $15 \%$. In his literature, he also compared and analyzed the individual revenue of the three mobile games on different platforms, as well as the overall revenue of the games-"Onmyoji", "Legend", and "Honor of Kings". The average individual income of Honor of Kings is much lower than that of Legend, but in terms of overall revenue, the honor of Kings is much higher for both of them. In 2016, The total revenue of Honor of Kings reached 7.8 billion yuan, compared with 210 million yuan for Onmyoji and 130 million yuan for Legend. In the second part, we analyze the longitudinal comparison. The vertical comparison refers to comparing the form of the same thing in different periods to understand the process of development and change of things and reveal the law of development of things. By studying how the number of players of 
Honor of Kings has changed from year to year, from December 2016 to May 2017 alone, Honor of Kings grew from 120 million users to 201 million [6]. By 2021, Honor of Kings has more than 600 million users, and the number is growing every day. This suggests that with such a large base of players, whatever Honor of Kings is doing will pay off.

\subsection{Literature research}

The literature search is a systematic and well-organized search from the already published data to identify a breadth of good quality references on a specific topic [7]. This article referred to some documents that mentioned disadvantages in marketing strategies.

First of all, we did some reading research. After knowing that mobile online games have occupied most people's lives, we decided to establish mobile online games as our research object. Then, by reading some past literatures, we noticed that using data is the best way to analyze the current situation in the mobile online game market. Moreover, we referred to some of the scholars' literature to analyze strategies in marketing.

\subsection{Case Study}

The case study is a research methodology that has commonly used in social sciences. It is based on an indepth investigation of a single individual, group or event to explore the causes of underlying principles [8]. This article uses "Honors of Kings" as an example to analyze strategies on online game marketing and some specific discussion of its current strategies. Using a typical example, it is easier to conclude the whole mobile online game market.

\section{RESULT}

\subsection{The development status of the online mobile game industry}

\subsubsection{Scale of development}

With the rapid development of science and technology to promote the continuous update of mobile phone functions, mobile phone games have become the public entertainment essential. According to Fu's survey, China's mobile games only started to develop on a large scale in 2013, which is relatively late compared to foreign markets. Nevertheless, in 2014, the mobile gaming market was filled with games of all kinds, with $\$ 2$ billion in revenue. The global mobile gaming market surpassed $\$ 3$ billion in 2015 and grew by more than 50\% in 2016 [9]. China has a large population and a solid consumer base to promote the development of the mobile game industry. Technological progress and Chinese policies also provide strong support for the market. At present, China's mobile gaming industry is a new industry. The use of mobile phone users and the market is expanding, with development prospects and a strong driving force.

\subsubsection{Game player group}

According to incomplete statistics in the mobile game market, Liu investigated that the current global mobile phone users have exceeded one billion. This vast number is still growing at high speed [10]. Compared with big games with high configuration on computers, mobile games are more attractive to potential customer groups due to their convenience and free time. Looking at the current mobile phone user information, we can see that the player group is mainly concentrated in young people around the age of 20. According to Liu pointed out in the article: $5 \%$ of users over 49 years old, $6 \%$ of users aged $36-40,13 \%$ of $31-35$ years old, $27 \%$ of users aged $26-30$, of which $41 \%$ are $20-25$ years old. $8 \%$ under the age of 20 [11]. This shows that most of the user age group is concentrated between young and middle-aged.

\subsection{The current state of marketing in the Mobile Online Games industry -- King of Glory as an example}

\subsubsection{The status quo of Product Strategy}

In August 2015, the mobile game Honor of Kings officially opened the test and entered the mobile Internet mobile game market with unstoppable momentum. Up to now, relying on the platform advantages of Tencent Group, its own high-quality products, precise brand positioning and marketing strategy, it has become the leader of the domestic mobile Internet mobile game market in just 4 years and has gained a large market share. The success of Honor of Kings, especially in brand building and marketing strategy, has important reference value for developing similar games.

\subsubsection{Pricing strategy based on consumer psy- chology}

According to Xu's study, Honor of Kings is implementing a pricing strategy based on consumer psychology. First of all, the price of the game props in king of glory is generally not high, and the price of all kinds of game props is basically within 100RMB. Although the price is not high but based on the hundreds of millions of users of Tencent, the price of ordinary people may bring huge profits at the same time, the so-called many a little makes a big profit. For example, the skin of one of the game's characters, "zhao yun”, costs only 71RMB, but according to statistics, this skin alone has brought in 200 million yuan for the king of glory. Second, Honor of Kings uses multiple pricing methods to effectively retain users. The honor of Kings is filled with free items that 
players need to log in daily or do other activities to collect. The giveaway of game props is conducive to encouraging the players to keep the heat of the game for a long time, thus improving the players' game experience, and these free game props will not bring a lot of burden to Tencent.

\subsubsection{Service marketing and platform selection}

According to incomplete statistics in the mobile game market, most of the players investigatory prefer the comfortable and convenient services they have offered. The success of Honor of Kings is based on its exquisite and rich game screen and game content. It always implements the principle of serving players and providing a comfortable game experience, and gives full play to the advantages of its relying party Tencent platform, which improves the coverage of its products and the coverage scope, and forms a unique marketing and promotion method. In Neil Boden's 4P marketing theory, the channel is also an important factor affecting marketing, and Tencent's platform itself meets all its advantages. According to a survey, Tencent QQ online users exceeded 270 million, and the total number of registered users in China exceeded 500 million. Then based on The QQ platform launched the use of WeChat, the user also broke through the 200 million marks. King of Glory has push messages on these platforms, and currently, most consumers learn about the game through online information, thus acquiring a considerable number of game users.

\subsubsection{Holiday discounts and promotions}

According to Xu's study, participating in Honor of Kings can get discounts of different degrees. For example, in the special days of Double 11, there will be a limited time activity skin, which only needs 1 RMB to buy, thus greatly enhancing players' purchasing power. Every holiday, there will be promotions and discounts in the game. For example, there will be a small discount on the skin that has no discount in daily life in the game. There will also be a limited time discount on heroes/skins every month. In some large events such as the anniversary, there will be a certain opportunity to go out of the mystery shop, new skin, skin return, star gifts, all heroes limited time free activities. All of these are good examples of the price advantage of your product, which can increase user engagement and keep players engaged for a long time.

\subsection{Problems with the marketing strategy of the Mobile Online Games industry}

\subsubsection{Downplaying the role of advertising}

According to Liu's article, advertising has been seen as one of the most important components in marketing. It has a huge effect on raising public attention and attracting potential customers. However, the game Honor of Kings barely has advertisements. Though Honor of Kings is now among the top 3 on the mobile download list, it still has the potential danger of losing customers because of making no advertisement [4].

\subsubsection{Inadequate punishment mechanism}

Though Honor of Kings has a player of over 600 million people in 2021 according to investment, it still faces losing customers because of the inadequate punishment mechanism [12]. According to Liu's article, the system of Honor of Kings has the problem of misjudging players [4]. For some players who hang up the game, the system cannot give proper or severe punishment as well. As a result, this led many players to feel disappointed and choose to give up this game, which reduced Tencent's revenue.

\subsubsection{Imperfection in-game experience}

The bug has been one of the unavoidable problems that can affect a player's game experience. This includes disappearance in background music, character's delay in movement, and unstable network environment. Those factors can influence players' emotion and experience ingame and cause leaves of loyal players.

\subsubsection{Bugs and weakness in the anti-addiction (teenager protection) system}

Although there are measures to ban minors from logging in after $10 \mathrm{pm}$ and to limit them to two hours before they are forced to log out, the system is still riddled with bugs. Many students use their parents' identities to evade the system, and there are numerous ways to crack it online. This reflects a lack of improvement in the system.

\section{DISCUSSION: OPTIMIZATION OF MARKETING STRATEGY IN THE ONLINE MOBILE GAME INDUSTRY}

\subsection{Increase online and offline promotion}

The rapid rise of Honor of Kings is due to the game's simple controls, which appeal to women who are not good at complicated controls and who want to try out the experience. Nevertheless, that is not to ignore the promotion of online platforms or offline events. As one of the most influential communication channels in the Internet era, live streaming platforms can attract several gamers for Honor of Kings. The honor of Kings can cooperate with live-streaming platforms and use professional players' events to attract the interest of high-end players. On the one hand, it increases the size of the game's audience. On the other hand, it can increase ordinary players' understanding and technical improvement for the game's operation. Live streaming platforms can also boost 
growth and exposure by signing up high-priced game anchors and hosting various events.

In addition to the live broadcast of the online platform, offline promotion and publicity activities cannot be ignored. For example, Honor of Kings could work with Tencent officials to organize a series of significant game tournaments in different cities, inviting some well-known professionals to serve as referees to attract players' participation and discussion. The game information of such a significant professional event will be discussed by users on social media, which can spread and influence to increase the visibility and exposure of the game. Besides, according to $\mathrm{Li}$, microblog is a powerful public opinion platform with the characteristics of openness, and the speed of information dissemination is fast. In this way, the website which can realize the rapid spread of public word of mouth can serve as the prominent occupying place for the online marketing of Honor of Kings, which can provide an open and professional interactive platform and an effective platform for game players to understand the game events and information announcements.

\subsection{Focusing on bug fixes and enriching game- play to improve the user experience}

For Honor of Kings, part of its success comes from its vivid scenes, cool heroes and special effects, and sound effects. The presence of bugs in a game can eat away at the player's patience and energy, leading to a loss of enthusiasm and interest in the game. To solve the "bug problem", mobile game programmers should increase their attention and time to fix bugs such as hero action delay, expand server capacity, deploy network adaptability, and improve the game experience [13].

The honor of Kings current gameplay is limited. The gameplay to competitive battle, after meeting the conditions, can participate in the game ranking. The basic game controls and gameplay are fixed, and there is nothing new. Mobile game developers need to focus on product diversification and innovation and explore more possibilities and new connotations that games can create. For example, focus on hot social topics that users are concerned about, and integrate them with gameplay to form high-quality game content. Mobile developers also need to break through and update their skills and controls, rather than just updating different heroes and skins; At the same time, mobile game developers can tap into China's rich history and culture, incorporate local culture into their games, actively promote traditional Chinese culture and bring novel gameplay accordingly [13].

\subsection{Strengthen game management and improve user experience}

In Honor of Kings, players often encounter bad behaviors from other players that can affect the self-game experience. This behavior includes teammates hanging up for too long, insulting other players with rude language and so on. However, its punishment is not perfect enough. Facing current defective punishment, stricter and improved penalties should be established to eliminate the game balance and affect the player's experience. Penalty points and warnings will be given to players who hang up or quit in the middle of one game. Some serious cases, such as players hanged up for more than half of the time in games or repeated the action of hanging up or quitting many times after warning, should be prohibited in recent games. At the same time, human supervisors can be added to warn and punish uncivilized phenomena more accurately and seriously to improve user trust and satisfaction.

\subsection{Improvement of anti-addiction system}

In the face of anti-addiction system's loopholes, developers should strengthen the management and supervision of the system. Developers should set up a stricter anti-addiction system to prevent teenagers from gaming too long and getting addicted. The system can improve by recognizing users' faces after playing online for a long time to enhance management. By doing so, the system can be severe and make real progress. At the same time, according to Zhan, developers should also accept the criticism and supervision of public opinion and constantly improve themselves [14].

\section{CONCLUSION}

\subsection{Summary main findings}

The honor of Kings itself is based on the Tencent platform for marketing, its use of marketing strategies has achieved good results, and for some of the shortcomings that have not been taken into account, it should use a complete method of strategy to improve product quality, expand the business map. In the development phase, effective access to the power of media on other platforms to develop can effectively improve the marketing effect. Consumption under the background of a new era, mobile games have to transform and upgrade products, how to make their dominance in this round of agitation. The honor of Kings and its team need to explore ideas, but no matter how to change, grasping user demand, improving product quality, rich content, improving the gaming experience, and exploring corporate social responsibility.

\subsection{Limitation and prospect}

This article uses secondary data, and there are some limitations. The accuracy is sometimes difficult to guarantee. Sometimes, the original data contains some errors and deviations, which the secondary analyst is unaware of, which may lead to deviation in conclusion. And secondary data is likely to be outdated data and sometimes cannot determine the reliability of secondary data used. 
In the subsequent survey and interview, we prospect used primary data. Because the primary data is authentic, reliable, targeted and time-sensitive, and the process of obtaining the data is controllable, it helps to improve the authenticity and reliability of the research results.

\section{REFERENCES}

[1] Yao Dan and Liu Shiyan, Analysis of marketing Strategy of Online Games in China -- Taking King of Glory as an example, China Academic Journal Electronic Publishing House, 2018(06), 201+203. DOI: http://www.cnki.net

[2] Li Tianjiao, Analysis of marketing strategy of mobile games -- Taking King of Glory as an example, China Academic Journal Electronic Publishing House, 2018, (02):125-129. DOI: http://www.cnki.net

[3] Chaffey, D and Ellis-Chadwick, F, Digital marketing: Strategy, Implementation and Practice. Pearson Education Limited. Harlow, 2012.

[4] Liu Yunfeng, On the marketing strategy of "King of Glory" mobile game and reference, China Academic Journal Electronic Publishing House, 2017(18): 313. DOI: http://www.cnki.net

[5] Xu Ke, Marketing strategy analysis of MOBA mobile game "Honor of Kings". China Academic Journal Electronic Publishing House, 2018. http://www.cnki.net

[6] Aurora Big Data: Honors of Kings research report https://www.jiguang.cn/reports/72

[7] NCBI, Literature search for research planning and identification of research problem. https://www.ncbi.nlm.nih.gov/pmc/articles/PMC5037943/

[8] Press Academia https://www.pressacademia.org/definition-of-case-study/

[9] Fu feng (2017) Analysis on the current situation and development trend of Mobile games in China, 2017(03):33-35. http://www.cnki.net

[10] Liu Tingting, Analysis of the current situation and development prospect of mobile game industry, 2018(19):360.

[11] Liu jin, Audience analysis of mobile media, The news of the world, 2015(09):126-128. DOI: http://www.qikan.com.cn

[12] Honor of Kings server when to increase the number of people, in 2019 Honor of Kings and peace elite user number, startup times and use time analysis: https://blog.csdn.net/weixin_34683262/article/details/119262663
[13] Zhan Wenxin and Hu Fuzhao, Research on the marketing innovation of "Honor of Kings" in the new consumption era, 2018 (06); 64-65. http://www.cnki.net

[14] Advantages and disadvantages of baidu library secondary data https://wenku.baidu.com/view/f77e5214866fb84ae 45c8d0a.html

[15] Sina, Advantages of first-hand information https://iask.sina.com.cn/b/6dWn4ENFZhH.html 